\title{
Preñez en ovejas Dohne Merino por inseminación artificial con dos dilutores y tiempos de refrigeración
}

\author{
Pregnancy in Dohne merino sheeps for artificial insemination with two diluters \\ and refrigeration times
}

\author{
${ }^{1}$ Miguel Guillén Portugal
}

\section{RESUMEN}

En la Estación Experimental Agraria Illpa, propiedad del Instituto Nacional de Investigación Agraria (INIA), se evaluó la preñez resultante de la inseminación artificial (IA) cervical con semen ovino, diluido y refrigerado a $5{ }^{\circ} \mathrm{C}$ con dos tipos de dilutores A (Tris) y B (Triladyl) a 0,24 y 48 horas en una dilución de 1:2 (semen/dilutor), y con dosis de $250 \times 10^{6}$ millones de espermatozoides por inseminación. Asimismo, se determinó las características seminales antes de cada inseminación. Ciento diecisiete ovejas primíparas y multíparas de la raza Dohne Merino fueron separados al azar en tres grupos de 39 individuos cada uno, a su vez por cada horario establecido ( 0,24 y 48 horas) se utilizó 13 animales. Todas fueron inseminadas de la siguiente manera: T1: semen fresco sin diluir (solo a 13 animales, ya que la mortalidad espermática era total a las 9 horas), T2: semen diluido en Triladyl (39 animales) y T3: semen diluido en Tris (39 animales). Los resultados de preñez y características seminales fueron analizados mediante la prueba de Chi-cuadrada y Tuckey, respectivamente. La preñez a 24 horas fue $B=69.2 \%(9 / 13)$ y con dilutor $A=46.2 \%(6 / 13)$. La preñez a 48 horas fue de $\mathrm{B}=30.8 \%(4 / 13)$ y $\mathrm{A}=46.2 \%(6 / 13)$. Los resultados evidenciaron que la preñez se ve afectada por el dilutor y tiempo de refrigeración a $5{ }^{\circ} \mathrm{C}$, mediante inseminación artificial con dosis de 250 millones de espermatozoides.

Palabras clave: Inseminación, motilidad, oveja, temperatura, viabilidad.

\section{ABSTRACT}

In the Agricultural Experimental Station "ILLPA", property of the National Institute of Agrarian Research (INIA), the pregnancy resulting from cervical artificial insemination (AI) with sheep semen, diluted and refrigerated at $5^{\circ} \mathrm{C}$ was evaluated with two types of diluents $\mathrm{A}$ (Tris) and $\mathrm{B}$ (Triladyl) at 0.24 and 48 hours, in a dilution of 1:2 (semen / dilutor), and doses of $250 \times 106$ million sperm by insemination. Likewise, the seminal characteristics were determined before each insemination. One hundred and seventeen primiparous and multiparous sheep of the Dohne Merino breed were randomly separated into three groups of 39 individuals each. Moreover, 13 animals were used for each established schedule ( 0.24 and 48 hours). Each sheep was inseminated, as follows: T1: undiluted fresh semen (only 13 animals, since sperm mortality was total at 9 o'clock), T2: semen diluted in Triladyl (39 animals) and T3: semen diluted in Tris (39 animals). The results of pregnancy and seminal characteristics were analyzed by the Chi-square test and Tuckey, respectively. The pregnancy at 24 hours was $B=69.2 \%(9 / 13)$ and with dilutor $A=46.2 \%$ (6/13); and the pregnancy at 48 hours was $B=30.8 \%(4 / 13)$ and $A=46.2 \%(6 / 13)$. The results showed that the pregnancy was affected by the dilutor and cooling time at $5^{\circ} \mathrm{C}$, by artificial insemination with doses of 250 million sperm.

Keywords: Viability, temperature, insemination, motility, sheep.

${ }^{1}$ Maestrante en Producción Animal y Reproducción Animal. Programa de Pós - Graduaçao em Zootecnia. Universidade Tecnologica Federal do Paraná. Estado de Paraná-Brasil. E-mail: miguelguillenportugalvet@gmail.com 


\section{INTRODUCCIÓN}

La inseminación artificial (IA) es un método importante en la producción ganadera, que permite difundir en la descendencia buenas características fenotípicas y genotípicas de un macho reproductor.

Las ovejas son poliéstricas estacionales. El ciclo estral es determinado por la estación del año y es influenciado por el fotoperiodo corto (otoño, invierno) (Gibbons, Cueto y García, 2007). En la región sur, el porcentaje de parición anual en ovinos fue de $41.4 \%$ en el año 2013, alcanzando la mayor actividad reproductiva en los meses de mayo-julio(MINAGRI, 2013).

Durante estos meses, los carneros utilizados como reproductores se ven sometidos a periodos de intensa actividad física, tolerando movimientos a diferentes predios y otras condiciones de estrés (trasporte, cambios de ambiente y alimentación, etc.). Por otro lado, existen riesgos físicos y sanitarios, que afectan la calidad seminal y ocasionan un bajo poder fecundante(Olivera, Gil, Gamarra y Fierro, 2011). Además, se pierde gran cantidad de espermatozoides en la monta natural, ya que la carga espermática excede la cantidad necesaria de espermios que se requiere para que la hembra quede preñada. Inclusive el carnero, en cada cubrición disminuye la cantidad del eyaculado y concentración espermática, provocando la reducción del porcentaje de fertilidad (Sepulveda, 2012). Desde que se iniciaron los primeros experimentos de la I.A. en ovinos, se utilizó semen fresco con resultados satisfactorios. Sin embargo, se comenzó a utilizar dilutores que, aparte de proteger el semen sin alterar sus características, aumentaron la dosis por inseminación, logrando abarcar una mayor cantidad de hembras por majada e incrementar la viabilidad de los espermatozoides a bajas temperaturas $\left(5^{\circ} \mathrm{C}\right)$ (Aisen, 2009b).

Dado el avance del uso de los dilutores, se realizaron estudios para evaluar, analizar, seleccionar y conservar las mejores características del material espermático, haciendo uso de diferentes parámetros evaluativos (motilidad individual progresiva, concentración espermática, integridad de membrana y vitalidad espermática), asegurando su calidad para la inseminación.

Con base a lo descrito, el presente trabajo tuvo como finalidad determinar la preñez comparando dos dilutores, uno comercial (Triladyl) y otro elaborado por medios caseros (Tris), realizando la inseminación en distintos horarios (24 y 48 horas), donde se evaluaron las características seminales a bajas temperaturas.

\section{MATERIAL Y MÉTODOS}

El trabajo se realizó en la Estación Experimental Agraria Illpa - INIA, ubicada en el distrito de Paucarcolla de la provincia de Puno, Región Puno. A $15^{\circ} 40^{\prime} 37^{\prime \prime}$ latitud sur y $70^{\circ} 04^{\prime} 38^{\prime \prime}$ longitud oeste, a una altitud promedio de $3815 \mathrm{~m}$, donde la temperatura fluctúa entre 1.60 a 16.30 ${ }^{\circ} \mathrm{C}$; con una media anual de $7{ }^{\circ} \mathrm{C}$ (CONCYTEC, 2007).

\section{Materiales}

\section{a. Material biológico}

Se utilizó 117 ovejas y 1 carnero de la raza Dohne Merino, además de 1 macho vasectomizado (retajo). Los ovinos fueron debidamente desparasitados con ivermectina al $1 \%$ (IBOMEC L.A. ( $)$, aplicada subcutáneamente (1 $\mathrm{ml} /$ animal).

Adicionalmente, se dosificó vía oral con albendazol al $12.5 \%$.

\section{b. Material empleado para la colecta de semen}

- Bolsitas de plástico en forma de cono para tubo colector.

- Tubo de metal para ovinos con escotilla de presión.

- Elásticos de jebe.

- Tubos Falcon (15 ml).

- Fundas de látex.

- Termo.

- Toalla para mantener la temperatura de vagina artificial.

- Cinta masking tape.

- Plumones de tinta indeleble.

\section{c. Material de laboratorio}

- Vórtex.

- Termómetro.

- Microscopio óptico.

- Platina calefactable.

- Láminas portaobjetos y cubreobjetos.

- Micropipeta graduada.

- Tubos de ensayo.

- Vaso de precipitado.

- Cámara Neubauer. 
- Jeringas hipodérmicas \#21, 23.

- Jeringa tuberculina (1 ml).

- Calentador.

- Hervidor eléctrico.

- Refrigeradora.

- Equipo de proctoscopía (proctoscopio y fuente de luz).

- Fundas de inseminación tipo pico de pato.

- Papel toalla.

- Estufa.

\section{Dilutores}

Se usó el dilutor Tris y Triladyl.

\section{a. Tris}

- Tris (hydroxymethyl aminomethano).

- Fructosa.

- Ácido cítrico 1-hidrato.

- Gentamicina-Estreptomicina.

- Yema de huevo.

- Agua bidestilada.

\section{b. Triladyl}

- Tris (hydroxymethyl aminomethano).

- Agua bidestilada.

- Glicerol.

- Ácido cítrico.

- Fructosa.

-Por cada $100 \mathrm{ml}$, se añadió los siguientes antibióticos: tilosina $5 \mathrm{mg}$, gentamicina 25 $\mathrm{mg}$, espectinomicina $30 \mathrm{mg}$ y lincomisina $15 \mathrm{mg}$.

Los reactivos utilizados en la tinción eosinanigrosina fueron:

- Eosina (soluble en agua).

- Nigrosina (soluble en agua).

- Tricitrato de sodio.

- Agua bidestilada.

\section{Método}

- Por cada $100 \mathrm{ml}$, se añadió los siguientes antibióticos: tilosina $5 \mathrm{mg}$, gentamicina 25 $\mathrm{mg}$, espectinomicina $30 \mathrm{mg}$ y lincomisina $15 \mathrm{mg}$. Los reactivos utilizados en la tinción eosinanigrosina fueron:

El método descrito respetó el código de ética aprobado para el uso de animales en experimentos científicos. En ese sentido, se seleccionaron 39 ovejas por cada tratamiento (T1, T2 y T3), las mismas fueron repartidas a su vez en grupos de 13 animales por cada horario ( $0,24 \mathrm{y} 48$ horas).

- Tratamiento 1 (T1): Fue el grupo control (semen fresco sin diluir). Se inseminó a las 0 horas y solo a 13 ovejas de las 39 , ya que hubo una mortalidad total de la muestra seminal a las 9 horas post colecta.

- Tratamiento 2 (T2): En este grupo se usó el dilutor Tris, inseminando 39 ovejas, las que fueron divididas en 3 horarios ( 0,24 y 48 horas). Fueron utilizados 13 animales por cada horario.

- Tratamiento 3 (T3): El semen fue diluido con el dilutor comercial Triladyl, inseminando 39 ovejas en celo, las cuales se distribuyeron en grupos de 13 por cada horario $(0,24$ y 48 horas).

\section{Preparación de los dilutores}

La preparación del dilutor Tris se realizó en forma mecánica con ayuda de una balanza digital previamente calibrada, y en el caso del dilutor Triladyl se realizó según las especificaciones del laboratorio(Minitube, 2010).

\section{d. Diluyente TRIS}

Para $10 \mathrm{ml}$ de dilutor:

$\begin{array}{lr}\text { - Tris buffer } & 0.36 \mathrm{~g} \\ \text { - Ácido cítrico } & 0.19 \mathrm{~g} \\ \text { - Fructosa } & 0.50 \mathrm{~g} \\ \text { - Yema de huevo } & 14 \% \\ \text { - Gentamicina } & 0.1 \mathrm{ml} \\ \text { - Agua bidestilada } & 7 \mathrm{ml}\end{array}$

\section{e. Diluyente TRILADYL}

Para $10 \mathrm{ml}$ de dilutor:

- Dilutor Triladyl 1 porción

- Yema de huevo 1 porción

- Agua bidestilada 3 porciones

Se diluye por unos minutos en el vórtex homogenizando la dilución.

\section{Colección de semen}

Se utilizó una hembra en celo para estimular la libido y la monta del carnero. El semen se colectó mediante la vagina artificial, ajustada a una temperatura de $55-60{ }^{\circ} \mathrm{C}$ y buena presión para el característico "golpe de riñón”. 


\section{Dilución de semen}

La dilución de semen se realizó en relación 1:2 (semen/dilutor)(Da Silva Maia, 2014). Se hizo de manera rápida para evitar el shock térmico. El dilutor, previamente preparado, fue puesto en un termo a baño maría a una temperatura de $37^{\circ} \mathrm{C}$ aproximadamente. Luego del eyaculado, fue extraído con una jeringa y añadido por la pared del tubo colector de acuerdo con el volumen de semen.

\section{Evaluación microscópica del semen a las 0, 24}

\section{y 48 horas}

\section{Motilidad individual progresiva}

Se calculó con un valor subjetivo mayor a 4 (con escala de $0-6$ ).

Tabla 1. Calidad de movimientos: Tipos

\begin{tabular}{cl} 
ESCALA & \multicolumn{1}{c}{$\begin{array}{c}\text { OBSERVACIÓN } \\
\text { (DESCRIPCIÓN) }\end{array}$} \\
& \multicolumn{1}{c}{$\begin{array}{c}\text { Espermatozoides sin movimiento } \\
\text { Espermatozoides con movimiento } \\
\text { pobre. Las cabezas de los } \\
\text { espermatozoides quedan fijas y } \\
\text { solo se mueven las colas, } \\
\text { pudiendo girar sobre sí mismos. }\end{array}$} \\
& $\begin{array}{l}\text { Espermatozoides sin movimiento. } \\
\text { Es per mato z o i d e s c o n } \\
\text { desplazamiento en círculos y } \\
\text { algunos progresivos. } \\
\text { Movimientos progresivos } \\
\text { sinuosos. } \\
\text { Movimientos progresivos } \\
\text { rápidos. } \\
\text { Movimientos progresivos muy } \\
\text { rápidos. }\end{array}$ \\
\end{tabular}

Fuente: Manual práctico para profesionales Biotecnología reproductiva - Inseminación artificial porcina (2007)

\section{Concentración espermática}

Se determinó mediante una fórmula validada (Hafez, 2000) el promedio del conteo de 5 cuadros que están ubicados en el centro de las dos retículas (superior/inferior) en la cámara de Neubauer, se multiplicó por 10000 y después por el volumen de la dilución.

\section{Concentración \\ $=$ prom. $\mathrm{x} 10000=$ núm. de espermios por $\mathrm{ml}$}

\section{Recuento espermático}

$=$ conc. $\mathrm{x}$ vol. de dilución $=$ núm. total de esp. en eyaculado

\section{Evaluación de vitalidad espermática}

Se colocó una gota de semen en una lámina portaobjeto y otra gota de coloración supravital eosina-nigrosina sobre la primera (Da Silva Maia, 2014). En un ángulo de $45^{\circ}$, se deslizó la muestra con otra lámina portaobjetos, procurando que la tinción sea uniforme y de película fina en toda la lámina. Se realizó el recuento de 200 espermatozoides, diferenciando vivos y muertos.

\section{Evaluación de integridad de membrana espermática (Hypoosmotic swelling Test)}

En un tubo de ensayo, se colocó $900 \mu \mathrm{l}$ de solución hiposmótica y $0.1 \mathrm{ml}$ de semen a una temperatura de $37^{\circ} \mathrm{C}$ por 1 hora para que ocurra la reacción (hinchazón de la cola); seguidamente, se le agregó $900 \mu \mathrm{l}$ de formol para detener la reacción(Aisen, 2009a). Se extrajo una gota que fue colocada en una lámina portaobjetos y visualizada en el microscopio óptico; se cuentan 100 espermatozoides.

\section{Refrigeración de semen}

La refrigeración se realizó en el tubo colector, descendiendo de una temperatura ambiente de 35 ${ }^{\circ} \mathrm{C}$ a $25-30{ }^{\circ} \mathrm{C}$, y posteriormente se bajó gradualmente en un refrigerador a $5{ }^{\circ} \mathrm{C}$ (Gibbons et al., 2007).

La dosis por inseminación se realizó de la siguiente manera:

\# Ovejas a I.A. $=\frac{\text { Conc.esp. } x \text { esp.vivos } x \text { esp.normales }}{250000000}$

Dosis/I.A. $=\frac{\text { Vol.total }}{\# \text { de ovejas }}$

\section{Inseminación artificial}

Para realizar la inseminación artificial, se apartó a las hembras en celo marcadas en la grupa por el retajo. Con ayuda de un operario, se suspendió a la 
hembra en forma adecuada. Una vez en posición, se limpió la vulva con papel toalla y se introdujo el proctoscopio en la vagina en un ángulo de $45^{\circ}$. Seguidamente, se adaptó la fuente de luz para la visualización de la cérvix y se introdujo la funda de inseminación con el semen ya cargado dentro de esta, procurando pasar la mayor cantidad de anillos cervicales (inseminación artificial vía cervical) depositando el semen en su interior.

\section{Determinación de fertilidad mediante ecografía a los 40 días}

Se utilizó un ecógrafo modelo ALOKA (5 mhz) vía transrectal para el diagnóstico de preñez a los 40 días post-inseminación, en este periodo el diagnóstico de gestación tiene una certeza muy alta $(95-100 \%)$.

\section{Análisis estadístico}

Los resultados de fertilidad y características seminales fueron analizados mediante la prueba de Chi-cuadrada en el programa SPSS V.19 y la prueba de Tuckey en el programa SAS (Statistical Analysis System) V.1999, respectivamente.

\section{RESULTADOS}

Tabla 2. Porcentaje de concentración espermática según dilutor y hora

\begin{tabular}{|c|c|c|c|c|}
\hline Dilutor & $\begin{array}{c}\text { Hora } \\
\text { de } \\
\text { análisis }\end{array}$ & $\mathbf{N}$ & $\mathrm{ml} \pm \mathrm{SD}$ & C.V \\
\hline Tris & 0 & 10 & $\begin{array}{l}3700 \times 10^{6} \pm \\
767028900^{\mathrm{a}}\end{array}$ & $28.92 \%$ \\
\hline Triladyl & 0 & 10 & $\begin{array}{r}3863 \times 10^{6} \pm \\
1117259047^{a}\end{array}$ & $20.73 \%$ \\
\hline
\end{tabular}

En la Tabla 2 se observa que la concentración espermática de la muestra seminal, es de 3863 x $10^{6}$ para el dilutor Triladyl y $3.7 \times 10^{6}$ para el dilutor Tris, no existe diferencia estadística entre ambos dilutores ( $\mathrm{p}>0.05)$.
Tabla 3. Porcentaje de motilidad individual progresiva, según dilutor y hora

\begin{tabular}{|c|c|c|c|c|}
\hline Dilutor & $\begin{array}{c}\text { Hora } \\
\text { de análisis }\end{array}$ & $\mathbf{N}$ & $\begin{array}{l}\text { Prom. } \\
\%\end{array}$ & $\begin{array}{l}\text { CV } \\
\%\end{array}$ \\
\hline \multirow[t]{3}{*}{ Tris } & 0 & 10 & $\begin{array}{l}5.20 \pm \\
0.79^{\mathrm{a}}\end{array}$ & 15.17 \\
\hline & 24 & 10 & $\begin{array}{l}3.60 \pm \\
0,99^{\mathrm{b}}\end{array}$ & 27.62 \\
\hline & 48 & 10 & $\begin{array}{l}2.55^{ \pm} \\
1.12^{\mathrm{b}}\end{array}$ & 43.80 \\
\hline \multirow[t]{3}{*}{ Triladyl } & 0 & 10 & $\begin{array}{l}5.40 \pm \\
0.52^{\mathrm{a}}\end{array}$ & 9.56 \\
\hline & 24 & 10 & $\begin{array}{l}3.70 \pm \\
0.48^{\mathrm{b}}\end{array}$ & 13.06 \\
\hline & 48 & 10 & $\begin{array}{l}2.80 \pm \\
0.48^{c}\end{array}$ & 17.25 \\
\hline
\end{tabular}

Letras diferentes $(a, b, c)$ en la misma columna, indican que hay diferencia significativa $(\mathrm{p}<0.05)$

En la Tabla 3 se observa que la motilidad individual progresiva (MIP) con dilutor Trisyema de huevo desciende por acción del tiempo de refrigeración. Donde a las 0 horas, se obtuvo el mayor porcentaje de MIP (5.20\%) a comparación del semen refrigerado a las 48 horas con un porcentaje de $2.55 \%$. El análisis estadístico indica que el factor tiempo de refrigeración ejerce una influencia significativa entre estos dos horarios $(\mathrm{p}<0.01)$. Resultados similares se obtuvieron con el dilutor Triladyl a las 0, 24 y 48 horas donde la motilidad individual progresiva desciende en un mayor porcentaje a las 48 horas $(2.80 \%)$ respecto a las 24 horas de refrigeración $(3.70 \%)(\mathrm{p}<0.01)$.

Tabla 4. Porcentaje de integridad de membrana por prueba de HOST, según dilutor y hora

\begin{tabular}{|lccccc|}
\hline Dilutor & $\begin{array}{c}\text { Hora } \\
\text { de } \\
\text { análisis }\end{array}$ & $\begin{array}{c}\text { Promedio } \\
\%\end{array}$ & $\begin{array}{c}\text { CV } \\
\%\end{array}$ & Mínimo / Máximo \\
\hline Tris & 0 & $71.50 \pm 3.95^{\mathrm{a}}$ & 5.53 & 65.00 & 77.00 \\
& 24 & $60.80 \pm 7.00^{\mathrm{b}}$ & 11.52 & 52.00 & 70.00 \\
\hline & 48 & $50.78 \pm 4.89^{\mathrm{c}}$ & 9.64 & 45.00 & 60.00 \\
\hline Triladyl & 0 & $72.80 \pm 5.59^{\mathrm{a}}$ & 7.68 & 64.00 & 82.00 \\
& 24 & $60.40 \pm 9.72^{\mathrm{b}}$ & 16.09 & 43.00 & 75.00 \\
& 48 & $37.80 \pm 8.68^{\mathrm{c}}$ & 22.95 & 23.00 & 52.00 \\
\hline
\end{tabular}


En la Tabla 4 se observa que el porcentaje de integridad de membrana, según la prueba de HOST, tuvo una reacción positiva decreciente a las 0,24 y 48 con el dilutor Tris siendo de $71.50 \%$, $60.80 \%$ y $50.78 \%$, respectivamente. El análisis estadístico indica que el factor tiempo de refrigeración tiene influencia significativa sobre el porcentaje de integridad de membrana $(p<0.01)$, siendo a las 0 horas de análisis donde se observó el mayor porcentaje de integridad de membrana. Resultados similares se obtuvieron con el dilutor Triladyl a las 0, 24 y 48 horas con $72.80 \%, 60.40 \%$ y $37.80 \%$, respectivamente; donde se evidencia que el tiempo de refrigeración a las 48 horas muestra el valor más bajo en comparación a las 24 horas de refrigeración $(\mathrm{p}<0.01)$.

Tabla 5. Porcentaje de vitalidad espermática, según dilutory hora

\begin{tabular}{|c|c|c|c|c|c|}
\hline Dilutor & $\begin{array}{l}\text { Hora de } \\
\text { análisis }\end{array}$ & $\mathbf{N}$ & Promedio \% & $\begin{array}{r}\mathrm{CV} \\
\%\end{array}$ & Mínimo \\
\hline Tris & 0 & 10 & $\begin{array}{c}64.25 \pm \\
3.45^{\mathrm{a}}\end{array}$ & 5.37 & 59.62 \\
\hline \multirow{5}{*}{ Triladyl } & 24 & 10 & $\begin{array}{c}53.45 \pm \\
5.59^{\mathrm{b}}\end{array}$ & 10.46 & 39.42 \\
\hline & 48 & 10 & $\begin{array}{c}45.84 \pm \\
4.11^{\mathrm{c}}\end{array}$ & 8.98 & 39.00 \\
\hline & 0 & 10 & $\begin{array}{c}65.11 \pm \\
2.89^{\mathrm{a}}\end{array}$ & 4.44 & 60.00 \\
\hline & 24 & 10 & $\begin{array}{c}56.52 \pm \\
2,65^{\mathrm{b}}\end{array}$ & 4.69 & 53.85 \\
\hline & 48 & 10 & $\begin{array}{c}37.23 \pm \\
5.93^{\mathrm{c}}\end{array}$ & 15.92 & 25.74 \\
\hline
\end{tabular}

La Tabla 5 muestra que la prueba de tinción supravital (eosina-nigrosina) en la vitalidad espermática, utilizando el dilutor elaborado a base de Tris-yema evaluado a las 0 horas, presenta el mayor porcentaje de espermatozoides vivos $(64.25 \%)$ en comparación con el semen refrigerado a las $24(53.45 \%)$ y 48 horas $(45.84$ $\%$ ), es decir, existe una influencia significativa del tiempo de refrigeración $(p<0.01)$. Un resultado similar se ha obtenido utilizando el dilutor comercial Triladyl; sin embargo, con este último, el porcentaje de vitalidad a las 48 horas de refrigeración fue mucho menor en comparación al dilutor Tris.

\section{Porcentaje de preñez}

Tabla 6. Diagnóstico de preñez con semen fresco, Tris y Triladyl a las 0 horas

\begin{tabular}{|c|c|c|c|c|}
\hline & \multicolumn{2}{|c|}{ Preñadas } & Vacías & Total \\
\hline \multirow{3}{*}{$\begin{array}{l}\text { FRESCO } \\
\text { 0 H. }\end{array}$} & $\begin{array}{l}\text { Frecuencia } \\
\text { observada }\end{array}$ & 10 & 3 & 13 \\
\hline & $\begin{array}{l}\text { Frecuencia } \\
\text { esperada }\end{array}$ & 8.5 & 4.5 & 13.0 \\
\hline & $\begin{array}{l}\% \text { de } \\
\text { FERT. }\end{array}$ & $76.9 \%$ & $23.1 \%$ & $100 \%$ \\
\hline \multirow{3}{*}{$\begin{array}{l}\text { TRIS } \\
\text { 0 H. }\end{array}$} & $\begin{array}{c}\text { Frecuencia } \\
\text { bservada }\end{array}$ & 7 & 6 & 13 \\
\hline & $\begin{array}{l}\text { Frecuencia } \\
\text { esperada }\end{array}$ & 8.5 & 4.5 & 13.0 \\
\hline & $\begin{array}{l}\% \text { de } \\
\text { FERT. }\end{array}$ & $53.8 \%$ & $46.2 \%$ & $100 \%$ \\
\hline \multirow{3}{*}{$\begin{array}{l}\text { TRILA } \\
\text { DYL } \\
0 \text { H. }\end{array}$} & $\begin{array}{l}\text { Frecuencia } \\
\text { observada }\end{array}$ & 9 & 4 & 13 \\
\hline & $\begin{array}{l}\text { Frecuencia } \\
\text { esperada }\end{array}$ & 9.5 & 3.5 & 13.0 \\
\hline & $\begin{array}{l}\% \text { de } \\
\text { FERT. }\end{array}$ & $69.2 \%$ & $30.8 \%$ & $100 \%$ \\
\hline
\end{tabular}

En la Tabla 6 se observan los resultados del diagnóstico de preñez a los 40 días post inseminación artificial. A las 0 horas, con semen fresco, dilutor Tris y dilutor Triladyl, se observa un porcentaje de $76.9 \%, 53.8 \%$ y $69.2 \%$, respectivamente. La prueba de Chi-cuadrada, indica que no hay asociación estadística entre el porcentaje de preñez y los tratamientos ( $p>0.05)$. Entonces, se puede entender que al añadir el dilutor Tris o dilutor Triladyl a un eyaculado e inseminar artificialmente por vía cervical a las 0 horas no incrementa significativamente el porcentaje de preñez en comparación con semen fresco sin diluir, al realizar el mismo método biotecnológico.

Tabla 7. Diagnóstico de preñez con semen diluido en Tris y Triladyl a las 0 horas

\begin{tabular}{|c|c|c|c|c|}
\hline & & Preñadas & Vacías & Total \\
\hline \multirow{3}{*}{$\begin{array}{l}\text { TRIS } \\
\text { O H. }\end{array}$} & $\begin{array}{l}\text { Frecuencia } \\
\text { observada }\end{array}$ & 7 & 6 & 13 \\
\hline & $\begin{array}{l}\text { observada } \\
\text { Frecuencia } \\
\text { esper ada }\end{array}$ & 8.0 & 5.0 & 13.0 \\
\hline & $\begin{array}{l}\% \text { de } \\
\text { FERT. }\end{array}$ & $53.8 \%$ & $46.2 \%$ & $100 \%$ \\
\hline
\end{tabular}




$\begin{array}{llccc}\text { TRILA } & \begin{array}{l}\text { Frecuencia } \\ \text { observada }\end{array} & 9 & 4 & 13 \\ \begin{array}{l}\text { DYL } \\ \text { O H }\end{array} & \begin{array}{l}\text { Frecuencia } \\ \text { esperada } \\ \text { \% de }\end{array} & 8.0 & 5.0 & 13.0 \\ & \text { FERT. } & \mathbf{6 9 . 2} \% & \mathbf{3 0 . 8} \% & \mathbf{1 0 0} \% \\ & \begin{array}{l}\text { Frecuencia } \\ \text { observada }\end{array} & 16 & 10 & 26 \\ \text { TOTAL } & \begin{array}{l}\text { Frecuencia } \\ \text { esperada } \\ \text { \% de }\end{array} & 16.0 & 10.0 & 26.0 \\ & \text { FERT. } & \mathbf{6 1 . 5} \% & \mathbf{3 8 . 5} \% & \mathbf{1 0 0} \% \\ & & & \end{array}$

La Tabla 7 indica que los porcentajes de preñez obtenidos con el dilutor Tris yema de huevo y el dilutor comercial Triladyl, realizando inseminación artificial inmediatamente después de la dilución en las muestras colectadas a las 0 horas, fueron $53.8 \%$ y $69.2 \%$, respectivamente. El análisis de Chi-cuadrado indica que no hay asociación estadística entre el porcentaje de preñez y los tratamientos.

Tabla 8. Diagnóstico de preñez con semen diluido en Tris y Triladyl a las 24 horas

\begin{tabular}{|c|c|c|c|c|}
\hline & & Preñadas & Vacías & Total \\
\hline \multirow{3}{*}{$\begin{array}{l}\text { TRIS } \\
24 \mathrm{H} .\end{array}$} & $\begin{array}{l}\text { Frecuencia } \\
\text { observada }\end{array}$ & 6 & 7 & 13 \\
\hline & $\begin{array}{l}\text { Frecuencia } \\
\text { esperada }\end{array}$ & 7.5 & 5.5 & 13.0 \\
\hline & $\begin{array}{l}\% \text { de } \\
\text { FERT. }\end{array}$ & $46.2 \%$ & $53.8 \%$ & $100 \%$ \\
\hline \multirow{3}{*}{$\begin{array}{l}\text { TRILA } \\
\text { DYL } \\
24 \text { H. }\end{array}$} & $\begin{array}{l}\text { Frecuencia } \\
\text { observada }\end{array}$ & 9 & 4 & 13 \\
\hline & $\begin{array}{l}\text { Frecuencia } \\
\text { esperada }\end{array}$ & 7.5 & 5.5 & 13.0 \\
\hline & $\begin{array}{l}\% \text { de } \\
\text { FERT. }\end{array}$ & $69.2 \%$ & $30.8 \%$ & $100 \%$ \\
\hline \multirow{3}{*}{ TOTAL } & $\begin{array}{l}\text { Frecuencia } \\
\text { observada }\end{array}$ & 15 & 11 & 26 \\
\hline & $\begin{array}{l}\text { Frecuencia } \\
\text { esperada }\end{array}$ & 15.0 & 11.0 & 26.0 \\
\hline & $\begin{array}{l}\% \text { de } \\
\text { FERT. }\end{array}$ & $57.7 \%$ & $42.3 \%$ & $100 \%$ \\
\hline
\end{tabular}

La Tabla 8 indica, respectivamente, un $46.2 \%$ y $69.2 \%$ de preñez usando los dilutores Tris y Triladyl a la misma hora de refrigeración. El análisis de Chi-cuadrado indica que no hay asociación entre el porcentaje de preñez y los tratamientos utilizados.
Tabla 9. Diagnóstico de preñez con semen diluido en Tris y Triladyl a las 48 horas

\begin{tabular}{|c|c|c|c|c|}
\hline & & Preñadas & Vacías & Total \\
\hline \multirow{3}{*}{$\begin{array}{l}\text { TRIS } \\
48 \mathrm{H} .\end{array}$} & $\begin{array}{c}\text { Frecuencia } \\
\text { observada }\end{array}$ & 6 & 7 & 13 \\
\hline & $\begin{array}{c}\text { Frecuencia } \\
\text { esperada }\end{array}$ & 5.0 & 8.0 & 13.0 \\
\hline & $\begin{array}{l}\% \text { de } \\
\text { FERT. }\end{array}$ & $46.2 \%$ & $53.8 \%$ & $100.0 \%$ \\
\hline \multirow{3}{*}{$\begin{array}{c}\text { TRILA } \\
\text { DYL } \\
48 \text { H. }\end{array}$} & $\begin{array}{c}\text { Frecuencia } \\
\text { observada }\end{array}$ & 4 & 9 & 13 \\
\hline & $\begin{array}{c}\text { Frecuencia } \\
\text { esperada }\end{array}$ & 5.0 & 8.0 & 13.0 \\
\hline & $\begin{array}{l}\% \text { de } \\
\text { FERT. }\end{array}$ & $30.8 \%$ & $69.2 \%$ & $100.0 \%$ \\
\hline \multirow{3}{*}{ TOTAL } & $\begin{array}{c}\text { Frecuencia } \\
\text { observada }\end{array}$ & 10 & 16 & 26 \\
\hline & $\begin{array}{l}\text { Frecuencia } \\
\text { esperada }\end{array}$ & 10.0 & 16.0 & 26.0 \\
\hline & $\begin{array}{l}\% \text { de } \\
\text { FERT. }\end{array}$ & $38.5 \%$ & $61.5 \%$ & $100.0 \%$ \\
\hline
\end{tabular}

La Tabla 9 revela un $46.2 \%$ y $30.8 \%$ de preñez a los 40 días post inseminación artificial con semen diluido con Tris yema de huevo y Triladyl a las 48 horas de refrigeración. El análisis de Chicuadrado indica que no hay asociación estadística entre la tasa de preñez y los horarios de refrigeración $(p>0.05)$.

\section{DISCUSIÓN}

Los resultados obtenidos en el grupo control con semen fresco sin diluir fue de $76.9 \%$; siendo superior a resultados de preñez hallados por Naim, Cueto y Gibbons (2004), con porcentajes de 58-65 \%. Según Milczewski, Kozicki, Luz y Neves (2000), la reducción como el aumento de la $\mathrm{T}^{\mathrm{o}}$ y la manipulación de semen promueven una disminución en la motilidad y daños estructurales bioquímicos y funcionales en los espermatozoides. Esto quedó confirmado al observar que las características seminales (MIP, HOST y vitalidad) descienden y se comportan de manera similar con ambos dilutores a 24 horas de refrigeración. Al mismo horario, la tasa de preñez con dilutor Tris es de $46.2 \%$, siendo superior a los resultados encontrados por Menchaca, Pinczak y Queirolo (2005) con $34.5 \%$. Tal descenso es ratificado por Evans y Maxwell (1990), quienes señalan que, durante el proceso de refrigeración, la fertilidad disminuye entre $10 \%$ a $35 \%$ por día de almacenamiento. Por otro lado, la tasa de preñez con dilutor comercial Triladyl no se ve 
afectada a pesar del descenso de la calidad seminal; y esto posiblemente gracias al glicerol presente en su medio, que funciona como un protector eficiente hasta las 24 horas. A las 48 horas de refrigeración, se observa una caída altamente significativa tanto en la preñez (30.8\%) como en sus características evaluadas. Según Gao (1992), el proceso de congelación rápida y descongelación lenta implicaría daños causados por la formación de pequeños cristales de hielo, los cuales se pueden agrupar formando cristales grandes, que rompen la membrana espermática, esto también fue confirmado por Fahy Lilley, Linsdell y Douglas (1990), que indican, el efecto protector del glicerol de las membranas del espermatozoide durante la criopreservación, y su efecto tóxico sobre ellas.

\section{CONCLUSIONES}

El dilutor y tiempo de refrigeración influyen en el porcentaje de fertilidad en ovejas Dohne Merino. Los dilutores Tris y Triladyl tienen diferentes efectos sobre las características seminales a 24 y 48 horas de conservación.

La utilización del semen hasta las 48 horas de refrigeración con dilutor Tris, tiene buenos índices de preñez.

\section{REFERENCIAS BIBLIOGRÁFICAS}

Aisen, E. (2009a). Reproducción ovina y caprina. Buenos Aires, Argentina: Editorial Inter-Médica.

Aisen, E. (2009b). Reproducción ovina y caprina, exploración clínica. Buenos Aires, Argentina: Editorial Inter-Médica.

CONCYTEC. (2007). CIT Illpa Estación Experimental Illpa - Puno.

Da Silva Maia, M. (2014). Tecnología de semen e inseminacion artificial em caprinos. Acta Veterinaria Brasilica 8 (suppl. 2), 389-95.

Evans G. y Maxwell W. (1990). Inseminación artificial en ovejas y cabras. Zaragoza, España: Editorial Acribia.

Fahy, G., Lilley, T., Linsdell, H. y Douglas, J. (1990). Cryoprotectant toxicity and cryoprotectan toxcicity reduction. In search of molecular mechanisms. Cryobiology, 27(3), 247-68. Recuperado de https://www.ncbi.nlm .nih.gov/pubmed/2199153

Gao, D. (1992). Glycerol permeability of human spermatozoa and its activation-energy.
Cryobiology, 29(6), 657-67. Recuperado de https://www.ncbi.nlm .nih.gov/pubmed/1478095

Gibbons, A., Cueto, M. y García, J. (2007). Obtención, procesamiento y conservación del semen ovino. INTA Bariloche.

Hafez, E. (2000). Reproducción e inseminación artificial de animales. México: Interamericana - McGraw Hill.

Menchaca, A., Pinczak, A. y Queirolo, D. (2005). Storage of ram semen at $5{ }^{\circ} \mathrm{C}$ : Effects of preservation period and timed artificial insemination on pregnancy rate in ewes. Anim Reprod, 2 (3), 195198. Recuperado de http://animalreproduction.org/article/5b5a6088f77 $83717068 \mathrm{~b} 47 \mathrm{fb}$

Milczewski, V., Kozicki, L., Luz, S. y Neves, P. $(2000)$. Inseminação artificial intrauterina e cervical em ovelhas utilizando sêmen refrigerado. Archives of veterinary science, 5 (1), 35-39. Recuperado de https://revistas.ufpr.b $\mathrm{r} /$ veterinary/article/view/3883

MINAGRI. (2013). Cadena productiva de ovinos. Recuperado de http://agroaldia.mina gri.gob.pe/biblioteca/download/pdf/a groeconomia/agroeconomia_ovino.pd $\mathrm{f}$

Minitube. (2010). Manual Triladyl ${ }^{\circledR}$ diluyente de semen bovino. Minitube 49(0), 0-3. Recuperado de http://www.perulacte a.com/wpcontent/uploads/2012/09/DI LUYENTE-DE-SEMEN-BOVINOTRILADYL.pdf

Naim, P., Cueto, M. y Gibbons, A. (2004). Inseminación artificial a tiempo fijo con semen ovino refrigerado. Archivos de zootecnia, 58 (223), 435-440. Recuperado de https://docplayer.es/1 7794389-Inseminacion-artificial-atiempo-fijo-con-semen-ovinorefrigerado.html

Olivera, J., Gil, A., Gamarra, J. y Fierro, S. (2011). Preservación seminal y su uso en programas de inseminación artificial a tiempo fijo en ovinos. Seminario Internacional de Ovinos, Paysandú Uruguay.

Sepulveda, N. (2012). Inseminación artificial en ovinos. XVI congreso Venezolano de producción e industria animal. XVI Congreso. Venezuela. 